\title{
ON DISCOVERING ELECTROMAGNETIC EMISSION FROM NEUTRON STAR MERGERS: THE EARLY YEARS OF TWO GRAVITATIONAL WAVE DETECTORS
}

\author{
Mansi M. Kasliwal ${ }^{1}$ and Samaya NisSanke ${ }^{2}$ \\ ${ }^{1}$ The Observatories, Carnegie Institution for Science, 813 Santa Barbara Street, Pasadena, CA 91101, USA \\ 2 Theoretical Astrophysics, California Institute of Technology, Pasadena, CA 91125, USA \\ Received 2013 August 29; accepted 2014 May 28; published 2014 June 11
}

\begin{abstract}
We present the first simulation addressing the prospects of finding an electromagnetic (EM) counterpart to gravitational wave $(\mathrm{GW})$ detections during the early years of only two advanced detectors. The perils of such a search may have appeared insurmountable when considering the coarse ring-shaped GW localizations spanning thousands of square degrees using time-of-arrival information alone. Leveraging the amplitude and phase information of the predicted GW signal narrows the localization to arcs with a median area of only a few hundred square degrees, thereby making an EM search tractable. Based on the locations and orientations of the two LIGO detectors, we find that the GW sensitivity is limited to only two of the four sky quadrants. Thus, the rates of GW events with two interferometers is only $\approx 40 \%$ of the rate with three interferometers of similar sensitivity. Another important implication of the sky quadrant bias is that EM observatories in North America and Southern Africa would be able to systematically respond to GW triggers several hours sooner than Russia and Chile. Given the larger sky areas and the relative proximity of detected mergers, $1 \mathrm{~m}$ class telescopes with very wide-field cameras are well-positioned for the challenge of finding an EM counterpart. Identification of the EM counterpart amidst the larger numbers of false positives further underscores the importance of building a comprehensive catalog of foreground stellar sources, background active galactic nucleus and potential host galaxies in the local universe. This initial study is based on a small sample of 17 detected mergers; future works will expand this sample.
\end{abstract}

Key words: binaries: close - catalogs - gravitational waves - stars: neutron - surveys

Online-only material: color figures

\section{INTRODUCTION}

The advent of advanced ground-based interferometers this decade is expected to usher in the era of routine gravitational wave (GW) detection (Barish \& Weiss 1999; Sigg \& LIGO Scientific Collaboration 2008; Accadia et al. 2011; Somiya 2012). Binary neutron star (NS) mergers are anticipated to be amongst the most numerous and strongest GW sources (Abadie et al. 2010). NS mergers are predicted to produce neutron-rich outflows and emit electromagnetic (EM) radiation across many wavelengths and timescales as the ejected debris interacts with its environment—gamma (e.g., Eichler et al. 1989; Paczynski 1991; Narayan et al. 1992; Metzger \& Berger 2012), optical (e.g., Li \& Paczyński 1998; Kulkarni 2005; Metzger et al. 2010; Roberts et al. 2011; Piran et al. 2012; Rosswog 2013), infrared (e.g., Barnes \& Kasen 2013; Kasen et al. 2013; Tanaka \& Hotokezaka 2013; Grossman et al. 2013), and radio (e.g., Hansen \& Lyutikov 2001; Pshirkov \& Postnov 2010; Nakar \& Piran 2011).

The discovery and characterization of the EM counterparts to GW detections promises to unravel astrophysics in the strong field gravity regime. Moreover, such EM-GW events will serve as the litmus test for whether NS mergers are indeed the sites of $r$-process nucleosynthesis (and hence, responsible for producing half the elements heavier than iron including gold, platinum, and uranium; e.g., Lattimer \& Schramm 1976; Mathews \& Cowan 1990). The accompanying surge of excitement in preparation for this endeavor has been described as analogous to the "gold rush" (Kasliwal 2013).

In Nissanke et al. (2013, hereafter, Paper I), we undertook an extensive end-to-end simulation on how to identify the elusive EM counterpart of a GW detection of NS mergers. We started with simulated astrophysical populations of NS mergers, evaluated GW detectability, and considered three critical steps: (1) GW sky localization and distance measures using different worldwide networks of three to five GW interferometers, (2) subsequent EM detectability by a slew of multiwavelength telescopes, and (3) identification of the merger counterpart amongst a possible fog of astrophysical false-positive signatures. We showed how constructing $\mathrm{GW}$ volumes and local universe galaxy catalogs can help identify and reduce the number of false positives, thereby enabling a secure EM identification.

Paper I simulated mergers detected by a network of five (Net5) to three identical (Net3) GW interferometers. Previous EM counterpart searches were limited to triply coincident detections (Aasi et al. 2013b). However, given projected timescales for construction of advanced GW interferometers, it appears that the early years (and possibly the first detections) could be limited to a network of only two LIGO interferometers (Aasi et al. 2013a). In this Letter, we consider new observational challenges specific to a network of only two GW interferometers (Net2).

\section{GW METHOD: DETECTION AND SOURCE CHARACTERIZATION}

As detailed in Section 2 of Paper I, we construct an astrophysically motivated population of $4 \times 10^{4} \mathrm{NS}-\mathrm{NS}$ binaries out to a limiting redshift $z=0.5$. Parameters include binary masses, luminosity distance $D_{L}$, inclination angle to the observer's lineof-sight $\cos \iota, \mathrm{GW}$ polarization angle $\psi$, and sky position $\mathbf{n}$ (where $\hat{\mathbf{n}} \equiv(\theta, \phi)$ is the unit vector pointing to a binary on the sky from a fixed Earth coordinate system, $\theta$ is the colatitude, and $\phi$ is the longitude). We associate each binary with a random 
orientation and sky position, and distribute the mergers assuming a constant comoving volume density for $D_{L}>200 \mathrm{Mpc}$ or using a $B$-band luminosity galaxy catalog (Census of Local Universe; Kasliwal 2011) for $D_{L}<200 \mathrm{Mpc}$.

Next, we select the NS mergers that are detectable with only the two LIGO interferometers at positions $\mathbf{x}_{H}$ and $\mathbf{x}_{L}$ (the subscripts denote the Hanford and Livingston sites, hereafter LIGO-H and LIGO-L). GW detection and source characterization methods use optimum matched filtering between GW predictions and simulated detector streams (see Section 3 of Paper I for details). The measured GW strain $h_{M}$ at a particular detector $\mathbf{x}_{H}$ or $\mathbf{x}_{L}$ is the sum of the two GW polarizations, $h_{+}$and $h_{\times}$, each weighted by their antenna response functions $F_{+,[\mathbf{H} / \mathbf{L}]}$ and $F_{\times,[\mathbf{H} / \mathbf{L}]}$, and multiplied by a time-of-flight correction. The time delay of the signal between the detector and the coordinate origin is given by $\tau_{[\mathbf{H} / \mathbf{L}]} \sim-\hat{\mathbf{n}} \cdot \mathbf{x}_{[\mathbf{H} / \mathbf{L}]} / c$, where $c$ is the speed of light. $h_{+}$and $h_{\times}$are functions of $D_{L}, \cos \iota$, masses, and the GW frequency $f$. The antenna responses, $F_{+,[\mathbf{H} / \mathbf{L}]}$ and $F_{\times,[\mathbf{H} / \mathbf{L}]}$, depend on $\hat{\mathbf{n}}$ and $\psi$. Based on triangulation with three or more interferometers, the time delay factor and phase effects dominate over amplitude when reconstructing sky location errors for the majority of sources (Nissanke et al. 2011; Veitch et al. 2012; Grover et al. 2014; Sidery et al. 2014; Rodriguez et al. 2014).

For LIGO-H and LIGO-L, we assume two anticipated noise curves at mid- and full-sensitivity (the upper red and black lines in Figure 1 of Aasi et al. 2013a) and idealized noise. We define a binary to be GW detectable if its expected signal-to-noise ratio $(\mathrm{S} / \mathrm{N})$ at each detector is $>6.5$ and its expected network $\mathrm{S} / \mathrm{N}$ (the rms of the individual S/Ns) $>12$ (Aasi et al. 2013a).

To infer the binary's sky position, we explicitly map out the full nine-dimensional posterior probability density function (PDF) using MCMC methods (see Section 3 of Paper I and Nissanke et al. 2010) and derive two-dimensional PDFs in $(\cos \theta, \phi)$. We took particular care to start each MCMC chain at random all sky positions and polarizations before marginalizing over the remaining seven-dimensional parameter space.

Finally, to better understand our MCMC derived measures, we also implement two toy models using amplitude-only GW waveforms. The first model incorporates only time-of-arrival information, whereas the second incorporates a combination of time-of-arrival and the detector antenna responses. Our second toy model assumes a six-dimensional GW waveform which incorporates time-of-arrival information weighted by an amplitude term of the form: $A_{F} \sim\left[F_{+}(\hat{\mathbf{n}}, \psi)\left(1+\cos ^{2} \iota\right) / D_{L}+\right.$ $\left.F_{\times}(\hat{\mathbf{n}}, \psi)\left(-2 \cos \iota / D_{L}\right)\right]$. By simulating hundreds of noise realizations, we map out the likelihood function for $(\cos \theta, \phi)$ for randomly orientated and located binaries on the sky at different $\mathrm{S} / \mathrm{Ns}_{[\mathbf{H} / \mathbf{L}]}$.

\section{GW RESULTS: DISTANCE, LOCALIZATION ARCS, AND SKY SENSITIVITY}

In Figure 1(a), we show the cumulative distance distributions of NS mergers detectable using only LIGO-H and LIGO-L at full sensitivity. As expected, the distance distribution of mergers detected by Net 2 is similar to those detected with Net 3 and Net5 in Section 2 of Paper I. At mid-sensitivity, the distance distribution is scaled down by a factor of $\sim 0.6$.

In Figure 1(b), we show the cumulative histogram of sky localizations at $95 \%$ confidence regions (c.r.) for Net2 and compare to Net3 and Net5 (Section 2 of Paper I). The median localization is $250 \mathrm{deg}^{2}$ compared with $17 \mathrm{deg}^{2}$ in Net3. As in Paper I, we expect NS black-hole (BH) binaries to show a distribution similar to NS-NS. At mid-sensitivity, we expect the

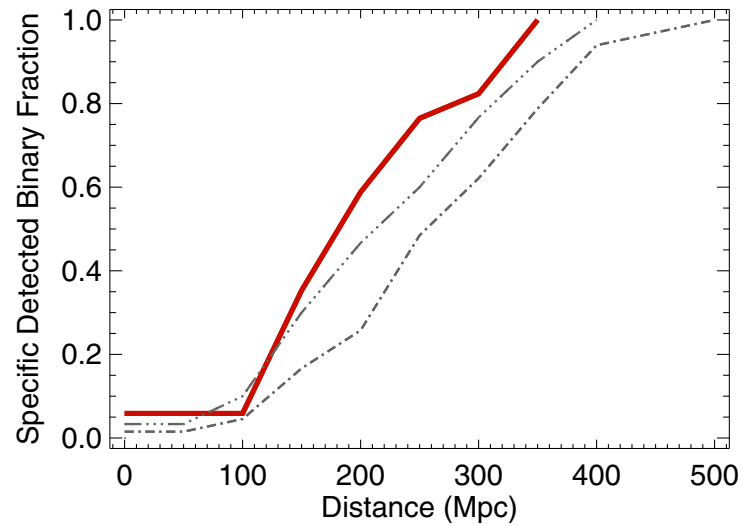

(a) NS-NS mergers: Luminosity Distance

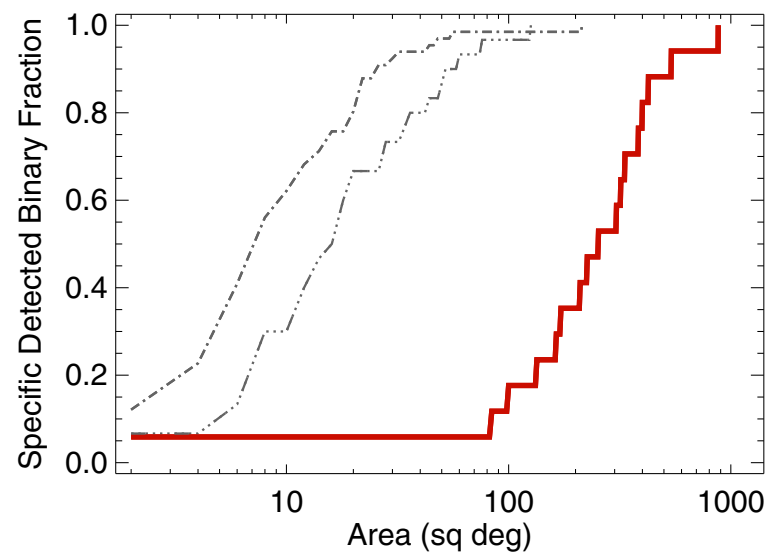

(b) NS-NS mergers: Sky errors

Figure 1. Cumulative distribution in luminosity distance (a) and $95 \%$ confidence sky error (b) of NS-NS mergers. Red lines denote a network of two GW interferometers. Gray lines denote Net3 and Net5 as presented in Paper I. We require an expected network $\mathrm{S} / \mathrm{N}>12$ and normalize to each specific network. (A color version of this figure is available in the online journal.)

specific distribution in sky localizations to be similar to those at full sensitivity because the majority of mergers will be detected at the $\mathrm{S} / \mathrm{N}$ threshold (distribution not shown here due to small number of detections).

In Figure 2, we show the localization shapes, orientation, and sky position of detected mergers at full sensitivity. Using only time of arrival of signals at LIGO-H and LIGO-L, sky localization estimates have so far predicted annular error rings for non-spinning mergers of several thousand $\operatorname{deg}^{2}$ (Aasi et al. 2013a). Instead, we find that inclusion of $F_{+}(\hat{\mathbf{n}}, \psi)$ and $F_{\times}(\hat{\mathbf{n}}, \psi)$ in the GW waveform's amplitude and phase information appears to significantly improve localization errors to arcs comprising several hundred $\mathrm{deg}^{2}$. For Net3-5, we found that degeneracies between parameters result in non-contiguous areas for a handful of mergers (e.g., Nissanke et al. 2011). Indeed, for a single spinning NS-BH merger using two initial LIGO sensitivities, Raymond et al. (2009) generated a localization arc by including the BH's spin.

The quadrupolar antenna patterns of LIGO-H and LIGO-L are $89 \%$ aligned. Figure 2 shows that Net 2 have significantly reduced sensitivity in two out of four sky quadrants for sources arriving in the plane of the interferometer arms. In contrast to Net3-5, we do not find a strong correlation between the $D_{L}$ and sky error as a result of the two-quadrant sky sensitivity. We find that two binaries at the same distance can have 


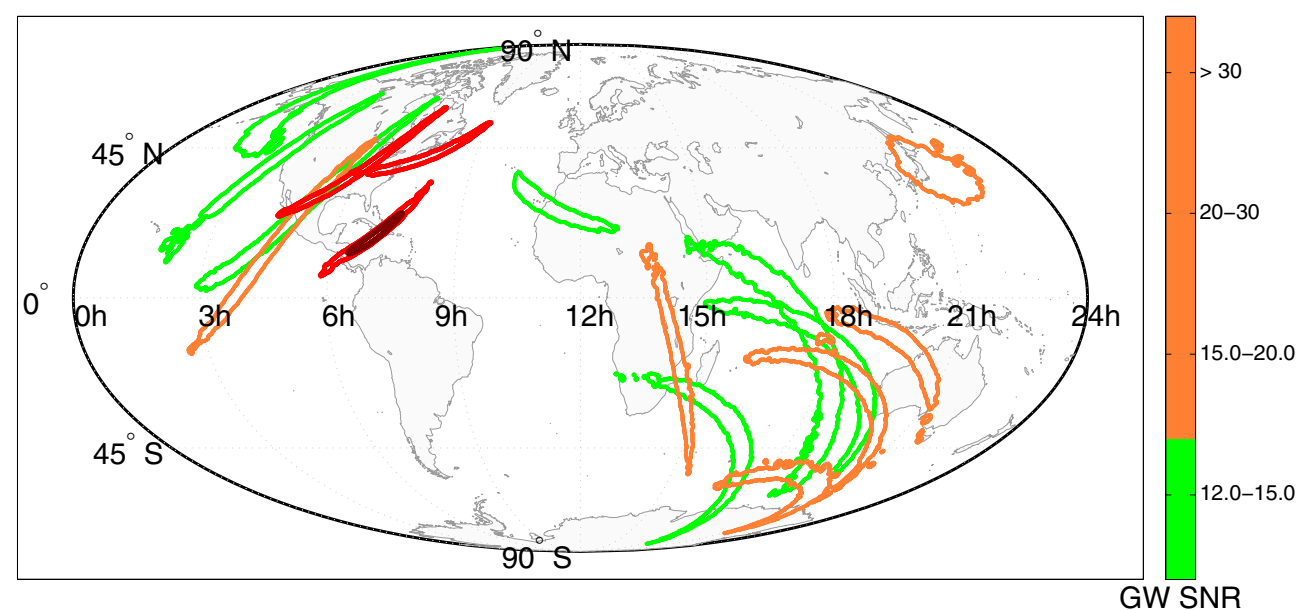

Figure 2. Sky location and localization arcs of mergers detected by LIGO-H and LIGO-L. Color represents expected network S/N. Note that the quadrupolar antenna pattern has a bias toward two sky quadrants. The rate of detected mergers is $\approx 40 \%$ of the rate of a three interferometer network. The EM observatory location dictates a time lag in response to GW trigger of up to one day (Table 1).

(A color version of this figure is available in the online journal.)

localization areas differing by an order of magnitude based on sky position.

Out of our underlying population, we find that only $17 \pm 4$ and $43 \pm 7$ mergers are detected in GWs using Net 2 and Net3, respectively. Therefore, Net 2 will detect $\approx 60 \%$ fewer mergers than Net3 using either $\mathrm{S} / \mathrm{N}$ threshold. Using NS merger rate estimates (Abadie et al. 2010) and Equation (7) in Paper I, we expect $0.3-490$ mergers annually for Net 2 . If co-moving volume is assumed, the rates are a factor of three lower.

We caution the reader that our results are based on an indepth study of a small sample. Hence, the median localization area presented here is uncertain by up to a factor of two. We note that multiple arcs are possible and were not seen in this particular study due to the assumed parameters of the small number of binaries. An extensive simulation of a thousand binaries is currently underway using both a low-latency algorithm (BAYESTAR) and MCMC parameter estimation adopting the 2015/2016 advanced LIGO sensitivity curves. Singer et al. (2014) find multiple localization islands such that about $15 \%$ of mergers have a probability peak in a location that is over a hundred degrees from the true location. We refer the reader to this upcoming work for a detailed study of the multiple localization islands in the early years.

\section{EM DETECTABILITY (TRIGGERED): RESPONSE-TIME, TILING, AND DEPTH}

Our GW results, indicating a sky quadrant bias and coarse arc-shaped localizations, present new challenges for triggered EM follow-up. (The challenge for contemporaneous, independent detection in the $\gamma$-rays or X-rays or low-frequency radio is unchanged.) Given the median localization of $250 \mathrm{deg}^{2}$ (at $95 \%$ c.r.), the tiling is currently beyond the scope of existing infrared, ultraviolet, and millimeter facilities. Hence, we consider follow-up by a representative set of optical facilities, with telescope apertures spanning 0.5-8 $\mathrm{m}$ and camera angles spanning 2-50 deg ${ }^{2}$ (see Table 1), and simulate relative detectability.

Due to the Net2 sky quadrant bias (Figure 2), mergers are preferentially detected overhead in the north and at hour angles around 12 in the south (relative to local sidereal time at LIGO-H/LIGO-L). Consequently, an EM observatory located around the same longitude as LIGO can respond instantly if

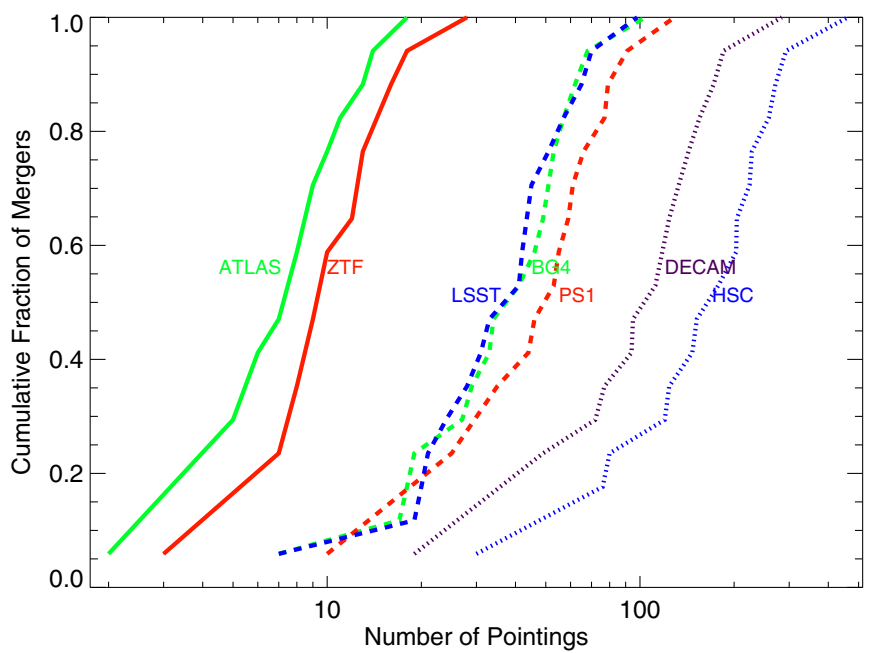

Figure 3. Cumulative distribution of number of pointings necessary to tile localization arcs at all sky positions. Color represents telescope diameter: $0.5 \mathrm{~m}$ class (green), $1 \mathrm{~m}$ class (red), $4 \mathrm{~m}$ class (purple), and $8 \mathrm{~m}$ class (blue). Line style represents camera angle: few tens of $\mathrm{deg}^{2}$ (solid), many $\mathrm{deg}^{2}$ (dashed), and few $\operatorname{deg}^{2}$ (dotted).

(A color version of this figure is available in the online journal.)

located in North America but only half a day later in Chile (see Table 1). This time-lag in response is critical for afterglows, which fade as a power law in time, and some kilonova models, which fade on few hours to day timescales. It is not relevant for radio facilities looking for late-time emission on the months to year timescale.

Due to the elongated arc-shape and the coarser localization of hundreds of $\mathrm{deg}^{2}$, tiling presents a major challenge. We compute an optimal tiling pattern to cover the GW localization contour (95\% c.r.) for each merger at each EM facility (Figure 3). While the widest cameras need $<20$ pointings, other facilities need hundreds of pointings. Naive division of the localization area by the camera field of view grossly underestimates the actual number of pointings required. This tiling inefficiency factor has a median value of 1.6 for BG4/HSC, 1.8 for DECAM, 2.0 for LSST/PS1, 2.6 for ATLAS/ZTF. The localization arcs have a median width of 6.5 (in agreement with time-of-arrival 
Table 1

Optical Facilities: Fraction of Detectable Kilonovae

\begin{tabular}{|c|c|c|c|c|c|c|c|}
\hline Facility & $\begin{array}{l}\text { Aperture } \\
(\mathrm{m})\end{array}$ & $\begin{array}{l}\text { Field of View } \\
\qquad\left(\operatorname{deg}^{2}\right)\end{array}$ & $\begin{array}{l}\text { Exposure } \\
\text { (s) }\end{array}$ & $\begin{array}{l}\text { Overhead } \\
\text { (s) }\end{array}$ & $\begin{array}{l}\text { Sensitivity } \\
(5 \sigma, i \mathrm{mag})\end{array}$ & $\begin{array}{c}\text { Detectable Fraction } \\
(-16 ;-14 ;-12 \mathrm{mag})\end{array}$ & $\begin{array}{l}\text { Lag } \\
\text { (hr) }\end{array}$ \\
\hline Palomar: Zwicky Transient Facility $(\mathrm{ZTF})^{\mathrm{a}}$ & 1.2 & 47 & 600 & 15 & 22.2 & $0.94 ; 0.35 ; 0.06$ & $1 \pm 2$ \\
\hline BlackGEM-4 (BG4) ${ }^{\mathrm{b}}$ & $4 \times 0.6$ & $4 \times 2$ & 600 & 15 & 22.2 & $0.65 ; 0.12 ; 0.06$ & $12 \pm 2$ \\
\hline Pan-STARRS1 $(\mathrm{PS} 1)^{\mathrm{c}}$ & 1.8 & 7.0 & 180 & 10 & 21.9 & $0.76 ; 0.18 ; 0.06$ & $3 \pm 2$ \\
\hline ATLAS-2 ${ }^{\mathrm{d}}$ & $2 \times 0.5$ & $2 \times 20$ & 600 & 5 & 21.0 & $0.65 ; 0.06 ; 0.06$ & $3 \pm 2$ \\
\hline CTIO: Dark Energy Camera (DECAM) ${ }^{\mathrm{e}}$ & 4.0 & 3.0 & 10 & 30 & 22.8 & $0.53 ; 0.47 ; 0.12$ & $12 \pm 2$ \\
\hline Subaru: HyperSuprimeCam (HSC) ${ }^{\mathrm{f}}$ & 8.2 & 1.77 & 1 & 20 & 22.4 & $0.47 ; 0.47 ; 0.18$ & $3 \pm 2$ \\
\hline Large Synoptic Survey Telescope (LSST) ${ }^{\mathrm{g}}$ & 8.4 & 9.6 & 1 & 2 & 22.4 & $1.00 ; 1.00 ; 0.65$ & $12 \pm 2$ \\
\hline
\end{tabular}

Notes.

a Kulkarni (2012) and E. Bellm (2013, private communication).

b P. Groot (2013, private communication), BlackGem plans up to 20 telescopes, see https://www.astro.ru.nl/wiki/research/blackgemarray.

$\mathrm{c}$ http://pan-starrs.ifa.hawaii.edu.

d J. Tonry (2013, private communication), ATLAS plans up to four units.

e D. DePoy (2013, private communication) and Bernstein et al. (2012).

${ }^{\mathrm{f}}$ http://www.naoj.org/cgi-bin/img_etc.cgi.

g LSST Science Collaborations (2009).

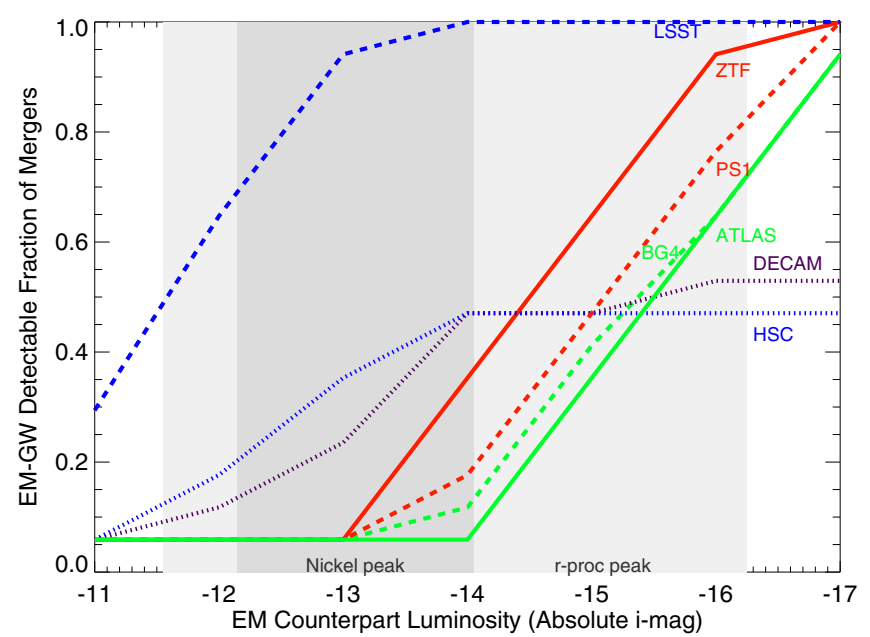

Figure 4. Fraction of mergers detectable by a given EM facility as a function of kilonova luminosity (expressed in absolute $i$-band AB magnitude). Color and line styles are same as in Figure 3. Shaded regions denote theoretical predictions for kilonovae (Barnes \& Kasen 2013; Kasen et al. 2013)—r-process powered peak (light gray; $M_{\text {ejecta }} \approx 10^{-1}-10^{-3} M_{\odot}, v_{\text {ejecta }} \approx 0.1 c-0.3 c$ ) and Nickel-56 powered peak (dark gray; $M_{\text {ejecta }} \approx 10^{-2}-10^{-3} M_{\odot}$ ). All fractions are relative as an accessibility window of $3 \mathrm{hr}$ with clear weather is assumed for all binaries at all facilities and no correction is made for lag in response (Table 1).

(A color version of this figure is available in the online journal.)

estimates, e.g., Fairhurst 2010). Narrow-angle cameras can tile more nimbly than wide-angle cameras (e.g., the BlackGEM-4 (BG4) tiling is $30 \%$ more efficient than the contiguous Pan-STARRS1 (PS1)).

With the number of pointings in-hand for each binary and for each EM facility, we compute the maximum exposure time (and hence, depth) allowable in a fixed duration. We assume three epochs (dithered to cover chip gaps) of $1 \mathrm{hr}$ each with a detection above $5 \sigma$ in at least two epochs as minimum criterion for EM detection. We take into account overhead between exposures which is dominated by readout for large mosaic cameras. Given the distance to each binary in our simulation, we convert the apparent magnitude depth to a luminosity.

Our detectability simulation results for Net2 are very different from those in Paper I for Net3-5. Figure 4 shows that small telescopes with large camera angles (e.g., Zwicky
Transient Facility (ZTF)) are more competitive than large telescopes with small camera angles (e.g., HyperSuprimeCam (HSC)) for detecting counterparts with an $i$-band luminosity brighter than $M_{i}=-14.5 \mathrm{mag}$. Recent tantalizing near-infrared excess observed in one short gamma-ray burst suggests bright kilonovae may be plausible (e.g., -15.6 mag in $H$-band; Tanvir et al. 2013). Note that response time is not folded into this figure as there is a diversity in kilonova models ranging from some that rise by 1 mag and others that decline by $1 \mathrm{mag}$ in the first $12 \mathrm{hr}$ (Barnes \& Kasen 2013).

Finally, EM follow-up is further hampered by weather, sunshine, moon-phase and visibility window. Thus, as discussed in Section 5.3 of Paper I, we emphasize that all detectable fractions presented here should be interpreted as relative.

\section{EM IDENTIFICATION: FALSE POSITIVES}

Optical detection of candidate EM counterparts in a single epoch is only the first step. Multiple epochs are essential to distill the true EM counterpart from thousands of astrophysical false positives in the foreground (e.g., moving objects in solar system, variable stars in Milky Way) and background (e.g., supernovae and active galactic nucleus (AGN) in higher redshift galaxies). An ongoing survey of the same sky location to a similar depth would provide a historic baseline of variability of unrelated sources and serve as a severe filter.

Timely identification of the EM counterpart is critical for obtaining spectroscopic and multi-wavelength follow-up before the transient fades. In Section 6 of Paper I, we considered five illustrative case studies to quantify the false-positive challenge and solutions in various scenarios. Here, we revisit the same five binaries in the context of Net2.

A beamed merger (391 Mpc). On account of the sky quadrant bias, this merger is not detected by Net 2 despite being beamed toward us. Given the lower rate of mergers detected with Net2 and the small fraction that is beamed $(<2.5 \%$ for opening-jet angles $<12^{\circ}$ ), we may not have the luxury of the relatively easier search for the EM counterpart of a beamed merger in the early years of Net2.

A close-in merger (69 Mpc). Net2 localizes this merger to $23 \mathrm{deg}^{2}$ at full sensitivity and $32 \mathrm{deg}^{2}$ at mid-sensitivity. This is a factor of $\approx 40-50$ coarser than Net3. Thus, the number of false positives would be proportionately larger and it is even 
more important to have a complete catalog of nearby galaxies. The fraction of "golden" binaries that are closer than $100 \mathrm{Mpc}$ remains $\approx 10 \%$.

A high Galactic latitude merger (139 Mpc). While Net3 localized this merger to $19.5 \mathrm{deg}^{2}$, Net2 localizes this to $223 \mathrm{deg}^{2}$ at full sensitivity (it is not detected at mid-sensitivity due to its distance). Therefore, there are 10 times more background sources and it is even more important to have a complete catalog of nearby galaxies and AGN variability.

A low Galactic latitude merger (125 Mpc). While Net3 localized this merger to $1.8 \mathrm{deg}^{2}$, Net2 localizes this to $100 \mathrm{deg}^{2}$ and $810 \mathrm{deg}^{2}$ at full and mid-sensitivity, respectively. Thus, the foreground is 55 times larger and it is even more important to build a catalog of stellar sources.

A Galaxy cluster merger (115 Mpc). On account of the sky quadrant location, this merger is not detected by Net 2 despite being relatively nearby.

\section{DISCUSSION}

The EM-GW challenge for NS mergers is three-fold: the GW localizations are wide (few hundred $\mathrm{deg}^{2}$ with two detectors) and the predicted EM counterparts are faint $\left(M_{i} \approx-12\right.$ to $-16 \mathrm{mag}$ ) and fast (few hours to few days). With LIGO-H and LIGO-L, we derive arc-shaped localizations with a median area of $250 \mathrm{deg}^{2}$ that are biased to only two sky quadrants. The rate of GW-detectable mergers is $\approx 40 \%$ of the rate of Net3 and the median localization area is 15 times coarser.

Strategies to maximize the odds of identifying faint and fast EM emission in wide GW arcs include the following.

1. A network of small $(<1 \mathrm{~m})$ telescopes, despite the shallow depth, can leverage observatory location and wide-field to maximize rapid response to find bright and fast-evolving EM emission. Given the GW sky quadrant bias, North America and Southern Africa are recommended as the best locations for rapid response to Net2 triggers.

2. A medium (1-3 m) telescope, despite the medium depth, can leverage an extremely large camera angle of few tens of $\mathrm{deg}^{2}$ to be best positioned for searching for EM counterparts brighter than $M_{i}<-14.5 \mathrm{mag}$. A dedicated facility with an ongoing survey to develop a baseline of historic variability is recommended.

3. A large $(>4 \mathrm{~m})$ telescope, even with a relatively narrow camera angle of few $\mathrm{deg}^{2}$, is uniquely positioned to find faint EM counterparts. A planned large time investment, facilitation of camera availability and minimization of overheads between pointings are recommended to be able to efficiently tile a larger fraction of mergers.

Independent of telescope size, the efficiency of a robust, real-time transient detection pipeline is an essential factor in assessing detectability. High-quality image subtraction requires a deep pre-explosion reference image of the same sky location, preferably taken with the same EM facility. Reliable candidate vetting needs a veteran machine learning algorithm, preferably trained on a large set of previous transient detections by the same EM facility. Thus, two facilities with identical hardware but disparate software would have different EM-GW detection capabilities.

Ongoing surveys are already successfully demonstrating the capability to discover optical transients which overcome the challenges of wide/faint/fast. For example, the discovery of multiple transients spanning kilonova luminosities addresses the faint characteristic (review in Kasliwal 2012) and the discovery of a relativistic explosion decaying on an hour timescale addresses the fast evolution (Cenko et al. 2013). Furthermore, the first two discoveries of optical afterglows in a $71 \mathrm{deg}^{2}$ error region (Singer et al. 2013; Kasliwal et al. 2013) bodes well to simultaneously address the fast, wide, and faint challenge.

In summary, the early years of a small number of coarse GW localizations will be challenging but tractable for an EM search. The combination of camera angle, telescope aperture, observatory location and survey software for each EM facility will delineate a different range in EM emission timescale and luminosity. A multi-pronged EM search would provide robust constraints on the vast phase space of kilonovae (ejecta mass, velocity, and composition). The findings of early searches will help plan EM-GW identifications to a larger number of better localized mergers in the era of three to five $\mathrm{GW}$ interferometers.

We thank C. Hirata and E. S. Phinney for careful reading of the manuscript. We acknowledge valuable discussions with E. Bellm, J. Bloom, P. Brady, Y. Chen, J. M. Désert, A. Georgieva, P. Groot, C. Galley, S. Mohta, L. Price, D. Reitze, L. Singer, and A. Weinstein. We thank D. Kasen for making kilonova models available. M.M.K. acknowledges generous support from the Hubble Fellowship and Carnegie-Princeton Fellowship. S.M.N. is supported by the David \& Lucile Packard Foundation.

\section{REFERENCES}

Abadie, J., Abbott, B. P., Abbott, R., et al. 2010, CQGra, 27, 173001 Accadia, T., Acernese, F., Antonucci, F., et al. 2011, CQGra, 28, 114002 Aasi, J., Abadie, J., Abbott, B. P., et al. (The LIGO Scientific Collaboration, the Virgo Collaboration) 2013a, arXiv:1304.0670

Aasi, J., Abadie, J., Abbott, B. P., et al. (The LIGO Scientific Collaboration and the Virgo Collaboration) 2013b, ApJ, 211, 7

Barish, B. C., \& Weiss, R. 1999, PhT, 52, 44

Barnes, J., \& Kasen, D. 2013, ApJ, 775, 18

Bernstein, J. P., Kessler, R., Kuhlmann, S., et al. 2012, ApJ, 753, 152

Cenko, S. B., Kulkarni, S. R., Horesh, A., et al. 2013, ApJ, 769, 130

Eichler, D., Livio, M., Piran, T., \& Schramm, D. N. 1989, Natur, 340, 126

Fairhurst, S. 2011, CQGra, 28, 105021

Grossman, D., Korobkin, O., Rosswog, S., \& Piran, T. 2013, MNRAS, 439, 757

Grover, K., Fairhurst, S., Farr, B. F., et al. 2014, PhRvD, 89, 042004

Hansen, B. M. S., \& Lyutikov, M. 2001, MNRAS, 322, 695

Kasen, D., Badnell, N. R., \& Barnes, J. 2013, ApJ, 774, 25

Kasliwal, M. M. 2011, PhD thesis, California Institute of Technology

Kasliwal, M. M. 2012, PASP, 29, 482

Kasliwal, M. M. 2013, Sci, 340, 555

Kasliwal, M. M., Singer, L. P., \& Cenko, S. B. 2013, GCN, 15324, 1

Kulkarni, S. R. 2005, arXiv:astro-ph/0510256

Kulkarni, S. R. 2012, arXiv:1202.2738

Lattimer, J. M., \& Schramm, D. N. 1976, ApJ, 210, 549

Li, L.-X., \& Paczyński, B. 1998, ApJL, 507, L59

LSST Science Collaborations, et al. 2009, arXiv:astro-ph/0912.0201

Mathews, G. J., \& Cowan, J. J. 1990, Natur, 345, 491

Metzger, B. D., \& Berger, E. 2012, ApJ, 746, 48

Metzger, B. D., Martnez-Pinedo, G., Darbha, S., et al. 2010, MNRAS, 406, 2650

Nakar, E., \& Piran, T. 2011, Natur, 478, 82

Narayan, R., Paczynski, B., \& Piran, T. 1992, ApJL, 395, L83

Nissanke, S., Holz, D. E., Hughes, S. A., Dalal, N., \& Sievers, J. L. 2010, ApJ, 725,496

Nissanke, S., Kasliwal, M., \& Georgieva, A. 2013, ApJ, 767, 124 (Paper I)

Nissanke, S., Sievers, J., Dalal, N., \& Holz, D. 2011, ApJ, 739, 99

Paczynski, B. 1991, AcA, 41, 257

Piran, T., Nakar, E., \& Rosswog, S. 2012, MNRAS, 430, 2121

Pshirkov, M. S., \& Postnov, K. A. 2010, Ap\&SS, 330, 13

Raymond, V., van der Sluys, M. V., Mandel, I., et al. 2009, CQGra, 26, 114007

Roberts, L. F., Kasen, D., Lee, W. H., \& Ramirez-Ruiz, E. 2011, ApJL, 736, L21 
Rodriguez, C. L., Farr, B., Raymond, V., et al. 2014, ApJ, 784, 119 Rosswog, S. 2013, RSPTA, 371, 20272

Sidery, T., Aylott, B., Christensen, N., et al. 2014, PhRvD, 89, 084060

Sigg, D. LIGO Scientific Collaboration. 2008, CQGra, 25, 114041

Singer, L. P., Cenko, S. B., Kasliwal, M. M., et al. 2013, ApJL, 776, L34
Singer, L. P., Price, L. R., Farr, B., et al. 2014, arXiv:1404.5623

Somiya, K. 2012, CQGra, 29, 124007

Tanaka, M., \& Hotokezaka, K. 2013, ApJ, 775, 113

Tanvir, N. R., Levan, A. J., Fruchter, A. S., et al. 2013, Natur, 500, 547

Veitch, J., Mandel, I., Aylott, B., et al. 2012, PhRvD, 85, 104045 